\title{
THE PARADOX OF GÖDEL'S NUMBERING AND THE PHILOSOPHY OF MODERN METAMATHEMATICS
}

\begin{abstract}
The author of this article critically analyses the proof of Gödel's famous theorem on the incompleteness of formalized arithmetic. It is shown that Gödel's formalization of meta-mathematics provides a proof of the incompleteness not of mathematical science but of the system of formalized meta-mathematics developed by Gödel himself. The arguments against the idea of the formalization of meta-mathematics are presented. The article suggests also an interpretation of the essence of mathematical truth. It is noted that the refutation of Gödel's proof does not suggest returning to Hilbert's program of formalism since the formalization of an axiomatic theory can't exclude the appearance of paradoxes within its framework. It is shown that the use of self-referential Gödel's numbering in a formalized system leads to the emergence of a Liar type paradox - a self-contradictory formula that demonstrates the inconsistency of that same system.
\end{abstract}

Keywords: formalized theories, metatheory, Gödel's theorem, Gödel's numbering, formalized metamathematics, inconsistency, paradoxes.

Introduction

Gödel's theorem on the incompleteness of formalized arithmetic is one of the most widely accepted and highly evaluated results of logical and mathematical thought of the 20th century (Gödel, 1931). It is this article, together with the previously proven fundamentally important theorem on the completeness of the first order predicate calculus that has determined the philosophy and ideology of all subsequent studies on the foundations of mathematics (Gödel, 1929). Encouraged by Gödel's results, many mathematicians began to investigate the consistency of formalized systems (Smoryński, 1977; Smullyan, 1991). Other researchers undertook an intensive study of the completeness of formalized calculi (Gentzen, 1935). And the most independent minds, despite general adoration before Gödel's proof of the incompleteness of formalized arithmetic, tried to restore the shaken authority of mathematical science by proving the completeness of formalized mathematical theories (Henkin, 1950; Hasenjaeger, 1953). The wave of publications on the significance of Gödel's theorems (Franzén, 2005), their philosophical interpretation (Rucker, 1995; Wang, 1997), the current demonstrative exposition (Smith, 2007), attempts to apply them far beyond the reasonable (Lucas, 1970; Feferman, 2011) continues to this day.

The first part of this article demonstrates that Gödel's proof of his incompleteness theo- 
rem is not valid, since in actuality it does not demonstrate the incompleteness of the Principia Mathematica and the related sufficiently rich systems.

The second part of this article discusses the general methodological problems of formalized theories and in its final section proves that if it is allowed to use Gödel's numbering in a system (including formalized axiomatic theories) then in this system one can build a Liar type paradox demonstrating the inconsistency of this system.

\section{Part I}

1.1. Young Kurt Gödel in his famous 1931 paper presented an instrument of logical proofs - Gödel's numbering - and constructed with its help an undecidable formula $\boldsymbol{G}$ (Gödel, 1931). Presuming that this formula belongs to the system of formalized arithmetic he concluded that the system of formalized arithmetic is incomplete (Gödel's theorem).

The principle point here is that in actuality Gödel's undecidable formula $G$ does not belong to the system of formalized arithmetic presented in Principia Mathematica (Rusell \& Whitehead, 1910) because to construct Gödel's formula $\boldsymbol{G}$ one needs Gödel's numbering which is absent in Principia Mathematica.

So we have to conclude that Gödel's proof of his incompleteness theorem is not valid, since it actually does not demonstrate the incompleteness of the Principia Mathematic $a$ and the related sufficiently rich systems.

1.2. On the other hand, if a mathematician would like to use Gödel's numbering in the system of formalized arithmetic he should do it by explicitly including Gödel's numbering in the scope of means of that formalized system
(Kleene, 1952). But in this kind enriched system one can build Gödel's undecidable formula $\boldsymbol{G}$ which would demonstrate that this kind enriched system of formalized arithmetic is incomplete. It should be underlined here that this proof of incompleteness relates to the system of formalized arithmetic enriched by $G \ddot{o}$ del's numbering, but not of the classical system of formalized arithmetic of Principia Mathematica.

\section{Part II}

The first part of this article concludes my refutation of Gödel's proof of the theorem on the incompleteness of formalized arithmetic. Dear readers, in the case you would like to object to my refutation, I ask you to apply only to the argumentation of the above presented first part of this article.

In the second part of this article, I present the general methodological considerations which help to demonstrate eventually that if it is allowed to use Gödel's numbering in a system (including formalized axiomatic theories) then one can build a Liar type paradox demonstrating the inconsistency of that system.

2.1. The consistency of axiomatic theories. An axiomatic theory is considered as a matter of serious discussion only after it is able to show solutions to the main problems of the given field of scientific knowledge. We call such a theory well-developed axiomatic theory. Just at this stage, the axiomatic method demonstrates its fundamental advantage. When a given axiomatic theory achieves such a weighty result - succeeds to solve the main problems of the field under investigation - one can express his suspicion in regard of this theory only in the 
case if he can present at least one fact (a true singular statement) that contradicts any axiom or theorem of this theory.

Until contradictory facts are found, a well-developed axiomatic theory is rightly regarded as a serious contender for the status of the true theory of the field under study. We emphasize that the theory achieves this high status precisely due to the successful axiomatic resolution of the problems of the field under study. Based on the presented analysis, we can propose the following preliminary definitions of the consistency and truth of axiomatic theories:

- An axiomatic theory is consistent, if it is in agreement with the facts of the field under study.

- An axiomatic theory is a serious pretender to the status of a true theory only insofar as it is successful in solving the main problems of the field under study.

(The expression "this theory is in agreement with the facts of the field under study" means that in this theory no statement is derived that is denied by any known fact of the field under study.)

2.2. True theory, absolute truth, and scientific progress. In connection with the proposed preliminary definitions, several questions arise. First, does an axiomatic theory remain for all time in the status of a "candidate" for becoming a true theory, or from a certain point in time and, according to a certain criterion, it deserves to be awarded the honorary title of a true theory? In a sense, it can be argued that in the eyes of the scientific community, theory achieves the status of "authentic truth" from the moment it is included in official university handbooks. This actual state of affairs necessarily assumes both the decades of successful activity of this theory in solving the important problems of the field under research, and its "impeccable behaviour" - complete absence of contradictory facts.

The second difficulty is expressed by the following question: "Are not the logic of Aristotle, the geometry of Euclid and the mechanics of Newton completely true and in this sense the ultimate and absolute truths of theoretical knowledge?" A negative answer to this question was established after the development and general recognition of the revolutionary theories of theoretical physics of the 20th century. Today, all educated people are well aware that the applicability of classical mechanics is limited to the field of phenomena of the macrocosm and is completely inapplicable neither to the world of atoms and elementary particles, nor to the world of sublight speeds. Formation of the algebra of logic in the 19th century and the victorious advance of mathematical logic in the 20th century are reasonably perceived as the emergence of logical systems more powerful than the Aristotelian syllogistic. And even the ideal of scientific knowledge - geometry - had to leave the pedestal of the absolutely true knowledge when mathematicians recognized the consistency of non-Euclidean systems of geometry developed in the 30s of the 19th century by Nikolai Lobachevski and János Bolyai, and especially when Bernhard Riemann presented to the society of mathematicians his grandiose system of geometries in 1854 (Riemann, 2004).

From what has been said, it can be concluded that there are no absolutely true general statements, laws and principles in the scope of scientific knowledge. And the fact that individual empirical judgments serve as 
the starting material of human theoretical knowledge leads to the conclusion that human cognition is not given either the knowledge of an absolute truth or a universal criterion for absolutely true general statements.

\subsection{The essence of mathematical truth.} In the modern formulation, this problem most often comes down to the question "How is truth defined in mathematics?". The complexity of the issue can be seen already in the fact that the author of the article "Truth" in Stanford Encyclopedia of Philosophy Michael Glanzberg was forced to turn to the help of 119 primary sources. The sub-section of this topic - "axiomatic theory of truth" - includes 15 different conceptions of truth. The state of affairs is extremely complicated by the fact that the problem of truth in its genesis and nature, without doubt, has an inextricable link with philosophy, where diversity and contradictory opinions are an unwritten norm. This article begins with the following paragraph: "Truth is one of the central subjects in philosophy. It is also one of the largest. Truth has been a topic of discussion in its own right for thousands of years. Moreover, a huge variety of issues in philosophy relate to truth, either by relying on theses about truth, or implying theses about truth. It would be impossible to survey all there is to say about truth in any coherent way" (Glanzberg, 2013).

The situation described does not inspire one more attempt to solve the problem of truth as a whole, and of the mathematical truth as its most difficult case, in particular. Yet let us make a new attempt by proceeding from the following premises:

- The problem of truth is a problem of all the sciences, and in this sense, a philosophical one.
- The universal definition of truth goes back to Aristotle: "Truth is the adequacy of the thought and reality." ("Veritas est adequatio ratio et rei")

- One needs to resolve specific questions of the theory of truth, and only then take up the development of a general conception.

- The central problem of the theory of truth in mathematics is expressed by the question: "What does exactly mean the notion reality in elucidating the truth of a mathematical statement?"

- The staircase of abstractions suggests the existence of a principle possibility for the reduction of any abstraction to the level of elementary ones. Apparently, this is confirmed by the reduction of all mathematics to the theory of sets.

- For the elementary abstractions, it is not difficult to find a correspondence with objects of reality.

In formalized theories, a central role is played by models - theories with simpler abstractions with strictly defined properties. With the help of models, one can answer the question of the correspondence of abstract (mathematical) statements to the real state of things.

Thus, an unambiguous conclusion follows from the principle of reducibility: the truth of any abstract (mathematical) statement is established by its correspondence to some simpler abstract model, which ultimately means its reducibility to a certain area of reality.

That is, the whole peculiarity of mathematical truth lies in the fact that in mathematics the truth of assertions is verified usually with the help of simpler abstract models which seem as if not having a direct relation to reality. Just this seeming separateness from 
reality creates a sense of paradox: on the one hand, the reliability of mathematical truths seems unshakeable and absolute, on the other hand, in view of the abstract nature of mathematical statements, they are perceived as completely detached from reality, and the question of their truth appears hanging in the air.

2.4. Formalism and formalization. According to Stefan Kleene's authoritative work "Introduction to Metamathematics", the formalization of a theory means a formal representation of the corresponding axiomatic theory (Kleene, 1952, chapter III). This is achieved in two steps. First, a complete symbolization of the axiom system of this theory is made. For this purpose, the initial symbols of the designation of all concepts appearing in the system of axioms should be fixed; with the help of this symbolism each axiom is presented as the certain initial formula. The second step of formalization is the fixation of the rules of inference, with the help of which only new formulas are obtained from the axiom system of the formalized theory. That is, thanks to formalization, the original axiomatic theory is transformed into a symbolic calculus. The philosophical strategy of constructing formalized calculi, originating from the works of Gottlob Frege and explicitly formulated by David Hilbert, is to achieve the greatest rigor in the formulation and development of axiomatic theories, which, in theory, should eliminate the emergence of contradictions and paradoxes in the developed formalized calculi. However, already on the eve of the publication of the second volume of his pioneering work "Grundgesetze der Mathematik", Frege was extremely disturbed by the letter of the young Bertrand Russell, in which the German scholar was informed of a para- dox, connected with the notion of the set of all sets, which was revealed in the first volume of Frege's work.

The discovery of similar paradoxes of the set theory and cardinal numbers, the BuraliForti paradox and the Cantor paradoxes, also belong to the same period. In the first decades of the twentieth century, they were supplemented by a popular analogue of Russell's paradox (the "paradox of barber"), while Richard revealed a paradox in the theory of real numbers. It was easy to notice that all these various paradoxes are very similar to the selfreferential ancient Liar paradox (Kleene, 1952, pp. 36-40).

This specific feature of almost all known logical-mathematical paradoxes suggests that the source of paradoxes is hidden rather in the self-referentiality of the corresponding concepts than in the axioms and logical means of inference. But the outstanding mathematician of the twentieth century, David Hilbert, conceived the idea of "completely eradicating" the paradoxes from the mathematical kingdom by building mathematical theories in the form of extremely strict formalized calculi. Thanks to Hilbert's authority and the fact that many paradoxes were perceived as belonging to the very foundation of mathematical knowledge, the direction of formalism in studies of the foundations of mathematics began to gain increasing recognition. This was facilitated by Kurt Gödel's proof of the completeness of the firstorder predicate calculus, the basis of the foundations of research on the foundations of mathematics (Gödel, 1929; Gödel, 1930; van Heijenoort, 1967). Naturally, the researchers had to cover the "gold rush" to be the first in proving the completeness of formalized arithmetic. But Gödel himself put the seemingly 
insurmountable barrier in front of the whole strategy of formalism by proving in 1931 his famous theorem on the incompleteness of formalized arithmetic.

\subsection{Is the idea of formalization of meta-} theories correct? Before we consider the methodological aspects of Gödel's theorem, we need to carefully consider the concept of metamathematics and its natural generalization the concept of metatheory. A formalized axiomatic system is called object-theory. Since the whole school of formalism arose to eliminate the possibility of a contradictions and paradoxes in mathematical sciences, it is natural that the primary task of the theory of formalized calculi was to establish their consistency. As the research of formalized theories has unfolded, an equally important possibility of establishing their completeness has emerged. These two principal qualities of formalized systems are studied by metatheory, which, naturally, should be outside the studied formalized system. Otherwise, the formalized theory would study itself, which is illogical. Moreover, if the metatheory is formalized, it will lose the sense of distinguishing between metatheory and object-theory.

But it was precisely the path of formalizing metamathematics that Gödel and his followers chose. True, in his famous article Gödel does not use the terms metatheory and $o b$ ject-theory, but by constructing his unsolvable formula $\mathrm{G}$ with the help of Gödel's numbering and recursive functions, he actually realizes the formalization of metamathematics. The overwhelming part of the excellent work of S. K. Kleene "Introduction to metamathematics" is devoted precisely to the strict and complete realization of Gödel's line of mathematization of metamathematics. In the litera- ture on foundations of mathematics, there is no objection to the evaluation of metatheory as proof theory - an idea originating from the work of D. Hilbert. Indeed, the only concrete predicate used in constructing Gödel's unsolvable formula $G$ is the predicate "to be a proof'.

However, Kleene, and apparently under his influence, many authors of articles on formalization and metatheory believe that formalized metatheory and metamathematics are part of mathematics. Klini insists that using Godel's enumeration, "metamathematics becomes a branch of number theory" (Klini, 1952, p. 205). The essence of the argument in favor of this position is this: mathematized metatheory is almost no different from other sections of mathematics and can quite be considered as a new section of mathematics. With this argument one could agree, if not for one important circumstance. In metamathematics in general, and in formalized metamathematics, in particular, no mathematical axiom or theorem is used, and no mathematical statement is derived from them. The whole content of metamathematical discussions, even after the formalization of metamathematics, is a question of consistency, completeness, solvability and similar characteristics directly related and even expressible with the predicate "to be a proof'. That is, after formalization, metatheory remains a tool for studying the demonstrative characteristics of formalized systems, including formalized mathematics. But how else could it be? After all, formalization changes only the language of presentation, but not the subject of research. Would the presentation of the Homer Iliad in the language of formalized mathematics enrich the mathematical sciences? And after the rhymed presentation of 
the Euclid Principles, would it lose its mathematical content?

At first glance, it may seem that there is not much difference whether to say "Formalized metamathematics is a part of mathematics" or to say "Formalized metamathematics is fully stated mathematically". In fact, the difference is fundamental. In the first case, the construction of Gödel's undecidable formula within the framework of formalized metamathematics speaks not only of the incompleteness of formalized metamathematics, but also of the mismatch of the mathematics itself, of which it is supposed to be a part. That is, assuming that the formalized metamathematics is part of mathematics, the undecidability of Gödel's formula can be represented as evidence of the incompleteness of mathematics itself.

In the light of modern meta-theoretical approach, it is of principle importance to distinguish mathematically stated theory of proof (meta-mathematics) from mathematics itself, even if the former is presented mathematically. Taking this differentiation for granted, the construction of Gödel's undecidable formula within the framework of formalized meta-mathematics will only demonstrate the incompleteness of formalized meta-mathematics, but not of the incompleteness of mathematics itself.

We again came to the sacramental question "Does Gödel's formula belong to mathematics or to the theory of proof?" As was proved above, the mathematization of any theory does not in any way change its content. This conclusion is even more convincing in the case of Gödel's formula. As Kleene explains, when interpreting the Gödel formula, it means a statement that "establishes its unprovability" (Klini, 1952, pp. 205; 207). Another interpreta- tion is simply impossible, for Gödel's formula contains only one concrete predicate "to be a proof." This unequivocally indicates that Gödel's formula belongs to the formalized theory of proof, and not to mathematics.

If the supporters of Gödel's proof of incompleteness paid sufficient attention to this circumstance and adequately interpreted it, then from the fact that Gödel's formula belongs to the formalized theory of proof they should conclude that just Gödel's formalized theory of proof (formalized meta-mathematics) is incomplete, and not the system of formalized arithmetic. That is, Gödel's theorem proves the incompleteness of the formalized theory of proof, but not of mathematics.

\subsection{Refuting Gödel'sproof does not mean} the necessity of vitalizing David Hilbert's program of formalization. Bad concepts remain bad concepts also after their formalization. For example, the concept of material implication of propositional logic is not adequate to the concept of logical inference and remains nonadequate also after the formalization in the frame of propositional calculus. It is rigorously proved that the propositional calculus is a consistent and complete formalized system. Yet the paradox of material implication is also well known.

Another example of a dubious instrument of argumentation is presented by self-reflexive expressions. In the context of this paper the most problematic instance of self-reflexivity is Gödel's numbering. This self-reflexive instrument was used in formalizing the system of metamathematics in his famous article on the incompleteness of formalized mathematics. All mathematicians knew about the affinity of selfreflexive expressions with the Liar paradox; nevertheless, they in the overwhelming majori- 
ty consider it permissible to use this very doubtful means.

The reason for such a protectionist attitude to the use of a very doubtful means is that without the use of self-reflective expressions, it becomes very difficult to construct certain important sections of modern mathematical science. After mentioning that the intuitive argument of Gödel's proof "skirts so close and yet misses a paradox" S. Kleene himself couldn't miss the feeling that proving a fundamental statement of incompleteness of formalized mathematic with the help of self-referential formula is rather undesirable taking into account the strictness of mathematical sciences. Apparently trying to justify the use of selfreferential numbering in Gödel's proof, S. Kleene writes: "Its proof, while exceedingly long and tedious in these terms, is not open to any objection which would not equally involve parts of traditional mathematics which have been held most secure" (Kleene, 1952, p. 206).

Suppose the position of mathematicians in relation to self-reflexive expressions can be understood. But how to go on with the philosophers and methodologists of science who have learned from mathematicians that there are used highly questionable self-reflexive expressions in the grandiose building of modern science?

2.7. Could the best logician of the twentieth century make a simple logical mistake? In the context of this article we have in mind the question, "Could Gödel miss that his formula, with its meaningful interpretation, does not express a mathematical statement?" After all, the interpretation of Gödel's formula $G$ means only one thing: "G asserts that it is unprovable". It does not take much mental effort to notice that this statement does not contain any mathematical content. How could an outstanding mathematician not notice this?

This could be facilitated by the following circumstances. Apparently, the main thing was that Gödel's theorem, elementary in its formulation, was proved by a grandiose and super complicated mathematical construction. It is quite natural that both its author and his followers should have the impression that this is just the proof of a mathematical statement. They could not have imagined that the grandiose and super-complex mathematical construction of Gödel's proof is only a rigorous representation of the self-reflective assertion that " $\mathrm{G}$ claims to be unprovable", which had only an indirect link with mathematics through the predicate "to be a proof" - the basic category of formal logic since the days of the Aristotelian Analytics.

The bias in Gödel's assessment of his formula as of a mathematical statement could be facilitated also by the fact that the young genius of mathematical science had the following psychological trait. From the school years, his each opinion the young genius considered the last truth, not subject to revision. His brother Rudolf Gödel mentioned that young Kurt had "a very individual and fixed opinion about everything and could hardly be convinced otherwise. Unfortunately he believed all his life that he was always right not only in mathematics" (O'Connor \& Robertson, 2003).

Unconventional progress in the study of school subjects could not but strengthen this trait of the character of the young Gödel. The most difficult tests of school education have always been associated with the assimilation of Latin grammar, and young Kurt managed to cope unerringly with this difficult matter. Rudolf Gödel had recalled later: "Even in High 
School my brother was somewhat more onesided than me and to the astonishment of his teachers and fellow pupils had mastered university mathematics by his final Gymnasium years. ... Mathematics and languages ranked well above literature and history. At the time it was rumoured that in the whole of his time at High School not only was his work in Latin always given the top marks but that he had made not a single grammatical error." (O'Connor \& Robertson, 2003).

\subsection{The paradox of Gödel's numbering.} Every reader familiar with the Liar paradox "L claims that L is false", on the first reading of the meaningful interpretation of Gödel's formula "G asserts that $\mathrm{G}$ is unprovable," notices the close relationship of Gödel's formula with the Liar paradox. Therefore, many researchers have a natural question, if can Gödel's selfreferential formula also lead to a paradox? Mathematicians reassure doubters that although the relationship to the paradox is close, but Gödel's formula does not raise the paradox, since here it is a matter of unprovability, and not falsity.

It would seem that the question of the paradox of Gödel's formula is in principle removed by Tarski's theorem. Alfred Tarski in 1936 proved that the arithmetical truth is indefinable in the system of formalized arithmetic (Tarski, 1936; Tarski, 1983). It would seem that mathematicians should have rejoiced after proving a theorem on the indefinability of truth. Indeed, if the truth is indefinable in the formalized theory, then it means that within the framework of this system there cannot arise either contradictions, or paradoxes. Tarski showed also that the concept of truth in this formalized theory can be determined by means of a richer formalized theory. Thus, the con- cept of truth in a system of formalized arithmetic constructed using the predicate calculus of the first order is not definable. However, the concept of truth of first-order formalized arithmetic can be defined in formalized arithmetic constructed by means of the predicate calculus of the second order, etc. Actually, we deal with a close analog of B. Russell's hierarchy of types (Bolander, 2017).

Here we digress from the essence of Tarski's theorem and confine ourselves to pointing out Tarski's those ideas that we need to demonstrate the fundamental assertion that the use of Gödel's numbering makes it possible to build a paradoxical (self-contradictory) formula in a system of formalized arithmetic. First, in the very definition of his system, Tarski directly indicates that one of its main elements is Gödel's numbering. Secondly, in his system of formalized arithmetic, he uses the predicate "to be true". Third, his proof of the theorem is based on the use of reductio ad absurdum, which makes it absolutely unessential to resort to the complex tool of recursive functions.

Tarski's theorem on the undefinability of truth directly concerns only a formalized theory. But Gödel's numbering can be used outside formalized theories too. This means that using the predicate "to be true" instead of the predicate "to be a proof", we can use Gödel's numbering to build in any system (including the formalized mathematics) the formula GT, which is a complete analogue of Gödel's formula G. For realizing this idea, it is sufficient to replace the predicate "to be a proof" by the predicate "to be true" in all steps of constructing Gödel's undecidable formula G. In the result of these procedures we get the formula GT which means "The proposition GT states that it is false." And then it is not difficult to come to 
the paradox: "The proposition GT is both true and false."

Indeed, let us turn to the help of the method reductio ad absurdum.

First, let us suppose that the proposition GT is true. Then, according to its content, the proposition GT must be false.

Now suppose the converse that the proposition GT is false. This will mean "double negation" - the falsity of asserting the falsity of the proposition GT. And then, according to the law of double negation, we have to conclude that the proposition GT must be true.

We come to a paradox, a demonstration of the self-contradiction of the proposition GT. The paradox of Gödel's numbering demonstrates the inconsistency of any system (including formalized mathematics) which allows using Gödel's numbering.

\section{Conclusions}

Gödel's proof of his incompleteness theorem is not valid, since in actuality it does not demonstrate the incompleteness of the Principia Mathematica and the related sufficiently rich systems.

If it is allowed to use Gödel's numbering in a system (including formalized axiomatic theories) then in this system one can easily build a Liar type paradox demonstrating the inconsistency of the system.

The mathematical genius of Kurt Gödel is unquestionable. Grandiose proofs of the theorem on the completeness of the first order predicate calculus and theorems on incompleteness of formalized arithmetic, he received at the age of up to 25 years. A few years later he received no less significant results, proving the consistency of systems using the most fun- damental means of set theory - the axiom of choice and the continuum hypothesis. The above demonstration of the inconsistency emerging from the use of Gödel's numbering in no way can reduce the merits of the great logician and mathematician in the history of the twentieth-century science.

\section{REFERENCES}

Bolander, T. (2017, August 31). Self-Reference. Retrieved November 20, 2017, from Stanford Encyclopedia of Philosophy:

https://plato.stanford.edu/entries/selfreference/.

Feferman, S. (2011). Gödel's Incompleteness Theorems, Free Will and Mathematical Thought. (R. Swinburne, Ed.) Free Will and Modern Science, 102122.

Franzén, T. (2005). Gödel's Theorem: An Incomplete Guide to its Use and Abuse. Wellesley: A. K. Peters.

Gentzen, G. (1935). Untersuchungen über das logische Schließen, I \& II. MathematischeZeitschrift, 39, 176-210, 405-431.

Glanzberg, M. (2013, January 22).Truth. Retrieved November 22, 2017, from Stanford Encyclopedia of Philosophy: https://plato.stanford.edu/archives/wi n2016/entries/truth/.

Gödel, K. (1929). Über die Vollständigkeit des Logikkalküls (Doctoral dissertation). University of Vienna.

Gödel, K. (1930). Die Vollständigkeit der Axiome des logischen Funktionenkalküls. Monatshefte für Mathematik, 
37(1), 349-360.

Gödel, K. (1931). Über formal unentscheidbare Sätze der Principia Mathematica und verwandter Systeme, I. Monatshefte für Mathematik und Physik, 38, 173-98.

Hasenjaeger, G. (1953). Eine Bemerkung zu Henkin's Beweis für die Vollständigkeit des Prädikatenkalküls der ersten Stufe. Journal of Symbolic Logic, $18,42-48$.

Henkin, L. (1950). Completeness in the Theory of Types. Journal of Symbolic Logic, 15(2), 81-91.

Kleene, S. C. (1952). Introduction to Metamathematics. North Holland.

Lucas, J. R. (1970). The Freedom of the Will. Oxford: Clarendon Press.

O'Connor, J. J., \& Robertson, E. F. (2003, October). Kurt Gödel. Retrieved November 19, 2017, from http://wwwhistory.mcs.stand.ac.uk /Biographies/Godel.html.

Riemann, B. (2004). Collected Papers. Heber City, UT: Kendrick Press.

Rucker, R. (1995). Infinity and the Mind: The Science and Philosophy of the Infinite. Princeton University Press.
Rusell, B., \& Whitehead, A. (1910). Principia Mathematica (Vol. I). Cambridge University Press.

Smith, P. (2007). An Introduction to Gödel's Theorems. Cambridge: Cambridge University Press.

Smoryński, C. (1977). The Incompleteness Theorems. In J. Barwise (Ed.) Handbook of Mathematical Logic (pp. 821-866). Amsterdam: North-Holland.

Smullyan, R. (1991). Gödel's Incompleteness Theorems. Oxford: Oxford University Press.

Tarski, A. (1936). Der Wahrheitsbegriff in den formalisierten Sprachen. Studia Philosophica, 1, 261-405.

Tarski, A. (1983).The Concept of Truth in Formalized Languages. In J. Corcoran (Ed.) Logic, Semantics, Metamathematics (pp. 152-278). Hackett.

van Heijenoort, J. (Ed.) (1967). From Frege to Gödel: A Source Book in Mathematical Logic, 1879-1931. Cambridge, MA: Harvard University Press.

Wang, H. (1997). A Logical Journey: From Gödel to Philosophy. MIT Press. 